\title{
Demonstration of gas detection based on MEMS suspended cantilever and on-chip optical read-out
}

\author{
Michal Zawierta ${ }^{1}$, Gino Putrino ${ }^{1}$, Matthew Myers ${ }^{2}$, Mariusz Martyniuk ${ }^{1}$, Adrian Keating $^{3}$, \\ K. K. M. B. Dilusha Silva ${ }^{1}$, Robert Woodward ${ }^{4}$, Roger Jeffery ${ }^{5}$, Lorenzo Faraone ${ }^{1}$, John Dell ${ }^{1}$ \\ ${ }^{1}$ School of Electrical, Electronic and Computer Engineering, \\ The University of Western Australia, Crawley, WA, Australia; \\ ${ }^{2}$ Hydrocarbon Detection Team, CSIRO, Australia; \\ ${ }^{3}$ School of Mechanical and Chemical Engineering, \\ The University of Western Australia, Crawley, WA, Australia; \\ ${ }^{4}$ School of Physics, The University of Western Australia, Crawley, WA, Australia \\ ${ }^{5}$ Panorama Synergy Ltd, Australia \\ Corresponding e-mail address: michal.zawierta@research.uwa.edu.au
}

\begin{abstract}
We demonstrate a toluene sensor based on the combination of integrated silicon photonics and microelectromechanical systems (MEMS). An integrated silicon photonics approach was used to create an optically resonant microcavity for monitoring the position of a cantilever. The demonstrated integrated cantilever read-out technology has the potential to provide physically robust chemical or biological sensors with picometer resolution for real world applications.
\end{abstract}

Key words: photonics, microelectromechanical systems, cantilever, sensors, toluene sensor

\begin{abstract}
Introduction
Microelectromechanical systems (MEMS) has established itself globally as a dominant sensing technology, providing commercialised sensing solutions for pressure, inertia, temperature as well as for more challenging applications in remote infrared imaging and energy harvesting [1]. However, to date MEMS has not delivered any commercially successful chemical or biological sensors, even though it has been established for some time that microcantilever-based sensing can detect chemical or biological agents at the level of single molecules [2] or zeptograms $\left(10^{-21} \mathrm{~g}\right)$ [3]. MEMS cantilever based sensors have demonstrated sufficient sensitivity to detect melanoma [4] or prostate cancer [5] and can be used to weigh biomolecules, single cells and single nanoparticles [6, 7]. The widespread application of this class of sensor has been largely impeded by the lack of a technology capable of performing high-resolution readout of large arrays of MEMS devices. We demonstrate a detection system based on MEMS suspended microcantilevers and an onchip optical read-out that has the potential to address large arrays.
\end{abstract}

Contemporary techniques for detecting cantilever motion or position include traditional
AFM based techniques (such as a laser beam reflected from the cantilever tip to a distant quadrant photodetector [8]), electrical sensing (such as piezoresistive, piezoelectric, capacitive, Lorentz force/emf sensing and tunnelling current techniques), optical sensing based on optical interference, or the use of diffraction from an optical grating formed by a line of cantilevers [3,9]. To monitor cantilever motion and/or position the optical methods (based on AFM read-out with quadrant photodetector) have high sensitivity but struggle to address large cantilever arrays, while the electrical methods (piezoresistive, piezoelectric or capacitive) are capable of addressing large arrays but are characterized by lower sensitivity [10]. The approach presented here combines the advantages of both [11].

Commercially available gas toluene sensors have significant limitations, among which the most important are complicated design and quite low sensitivity - minimum detectable level is typically around $1 \mathrm{ppm}$ [12], while it is often required to detect much lower concentrations of toluene. For example, Sick Building Syndrome (SBS) is a significant problem in Japan caused by volatile organic compounds and aldehydes, where the maximum acceptable toluene concentration is specified as 70 ppb [13]. Recent studies show significant negative 
influence of toluene exposures on living organisms [14, 15].

This work demonstrates a technique based on the combination of integrated silicon photonics and microelectromechanical systems (MEMS) to create an optically resonant microcavity for monitoring the position of microcantilevers. The technique is used to implement a toluene sensor.

\section{Sensing approach}

Our sensing and read-out approach is schematically shown in Fig. 1 and is based on the suspended cantilever modulating light transmission in an underlying optical waveguide in a manner similar to a tunable Fabry-Perot cavity. An optically resonant cavity is created when a microcantilever with a reflective undersurface is suspended above a Bragg grating etched into a waveguide. As light travels through the silicon waveguide, the grating will couple a proportion of the light out of the waveguide towards the microcantilever beam. The microcantilever will reflect the light back towards the grating, creating a resonant cavity. The optical resonance phenomena within the grating-cantilever cavity causes the re-coupling into the waveguide to be dependent on the grating-cantilever separation. Fig. 2 (a) shows finite-difference time-domain (FDTD) simulations of the optical field distribution between interrogating grating and cantilever with separation of $0.75 \mu \mathrm{m}$. Constructive interference takes place and maximizes the output power at this separation. Fig. 2 (b) shows the same device with a grating to cantilever separation of $1.25 \mu \mathrm{m}$. Destructive interference reduces output signal to minimum at this separation. Fig. 3 compares simulation and measurement results for suspended microcantilever as a function of the waveguidemicrocantilever gap [13].

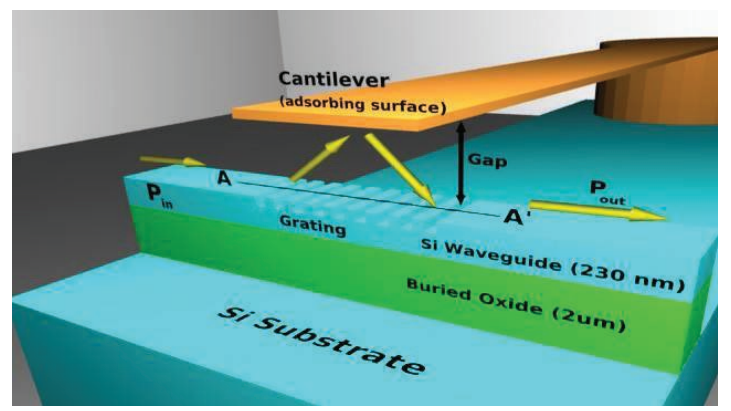

Fig. 1. Isometric view of the interrogating structure. Some of the light travelling through an underlying waveguide is allowed to diffract and reflect from the underside of the sensing cantilever back into the waveguide resulting in optical interference that is dependent on the waveguide-cantilever separation.
The intensity of the light re-coupled into the waveguide is amplitude modulated by movement of the MEMS microcantilever. This method has been demonstrated to sense cantilever position with picometer sensitivity [17]. As the diffraction grating and the microcantilever are mechanically connected via the chip substrate, common-mode noise is rejected, making this read-out technique extremely low noise and compact. The integrated nature eliminates mechanical/optical alignment issues, and enables scaling to large numbers of microcantilevers using wavelength, frequency and/or time division multiplexing.

The silicon photonics component was fabricated using a silicon-on-insulator (SOI) substrate via the ePIXfab program, on which silicon nitride cantilevers with a gold reflecting underlayer were fabricated using surface micromachining. Subsequently, the cantilever top surface was coated with a toluene functionalization layer of polyisobutylene (PIB) by using a drop casting technique. PIB

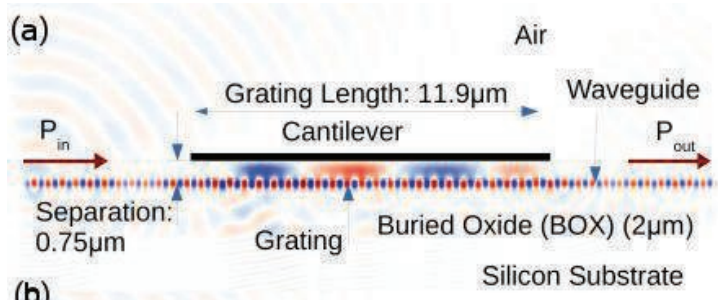

(b)

Silicon Substrate

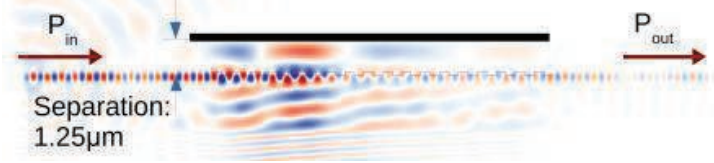

Fig. 2. The optical field distributions for propagated $1550 \mathrm{~nm}$ light simulated using FDTD modeling in MEEP when the cantilever is at the height required to create (a) constructive and (b) destructive interference [16].

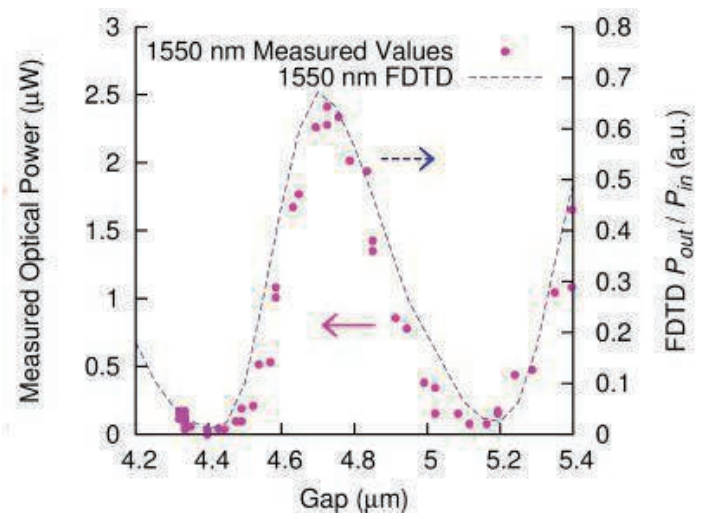

Fig. 3. Measured optical power transmitted through the device for two vibration cycles of a suspended cantilever (full circles) compared to 2D FDTD simulation results (dashed line) as a function of the waveguide-cantilever gap. 
expands/contracts with absorption/desorption of toluene and induces microcantilever bending [18].

Cantilever sensors can operate in static or dynamic mode. The static mode involves measurement of the cantilever deflection due to changes in functionalization layer parameters, while dynamic mode involves excitation of microcantilever vibration at mechanical resonant frequency and monitoring of the changes in frequency. This paper demonstrates the operation of a static mode microcantilever sensor.

\section{Sensor demonstration and outlook}

The experimental set-up used to expose the fabricated chip to toluene is shown in Fig. 4. Fiber optics and input/output gratings are used to couple $1550 \mathrm{~nm}$ light in/out of the chip. Initially, the fabricated sensor was placed in a test chamber filled with pure nitrogen. A small amount of toluene gas, diluted by nitrogen was introduced into the chamber and, after a period of time, the toluene flow was turned off and the chamber was filled with pure nitrogen again. Fig. 5 shows the amplitude of the optical signal transmitted through the device as a function of time, before, during, and after toluene exposure. A significant increase and subsequent saturation of the transmitted optical signal is clearly observable in Fig. 5 and is

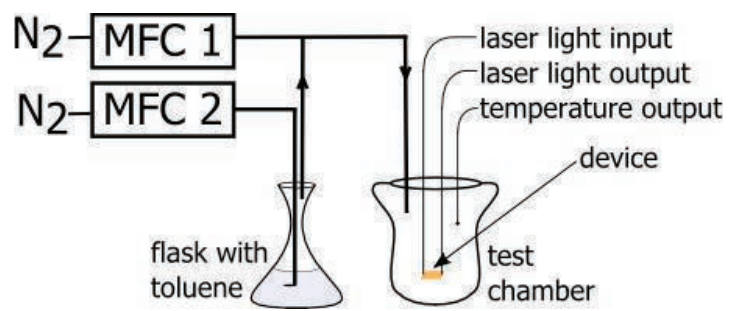

Fig. 4. Set-up of toluene detection experiment. Dilution nitrogen is delivered through mass flow controller (MFC 1) directly to the test chamber. During the toluene exposure period, additional nitrogen, controlled by a second mass flow controller (MFC 2) is passed through a bubbler in a flask with toluene and mixed with the main flow into the chamber.

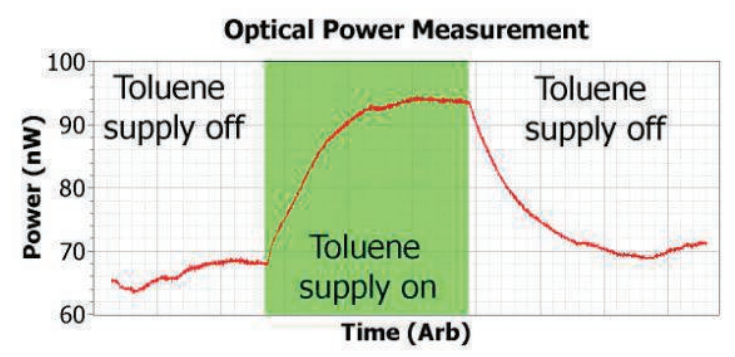

Fig. 5. Optical signal transmitted through the device as a function of time before, during, and after toluene exposure. associated with exposure to toluene. This behavior is reversed once the toluene source is turned off.

The ability to perform high-resolution monitoring of large arrays of MEMS devices will have a large impact on the capabilities of future commercial solutions. To address large arrays of sensors on a single chip various multiplexing techniques could be used. For example individual sensing cantilevers can be connected in a parallel optical circuit by coupling a set of waveguides to the output of an on-chip wavelength division demultiplexer and using a broadband or WDM source, allowing each waveguide containing a microcantilever to be interrogated using a separate wavelength. The output of all waveguides can be detected by separate detectors or combined in a second multiplexer and coupled to an output fiber for subsequent off-chip processing. This technique is suitable for both static and dynamic mode of operation.

For an array implementation of sensing microcantilevers operating in dynamic mode, frequency division multiplexing can be utilized by arranging several microcantilevers of different length along the same waveguide with each cantilever above its own grating. A schematic of how this system could operate is shown in Fig. 6. The resonant frequency of each microcantilever could be separately detected to track analyte adsorption on each microcantilever. Alternatively, electrostatic actuation of each microcantilever allows time division multiplexing to be used by selectively resonating each sensor. A combination of these techniques could be used to further increase microcantilever density.

\section{Conclusion}

We have demonstrated a toluene sensor based on the combination of integrated silicon photonics and microelectromechanical systems (MEMS) to create an optically resonant microcavity for monitoring of the position of cantilevers.

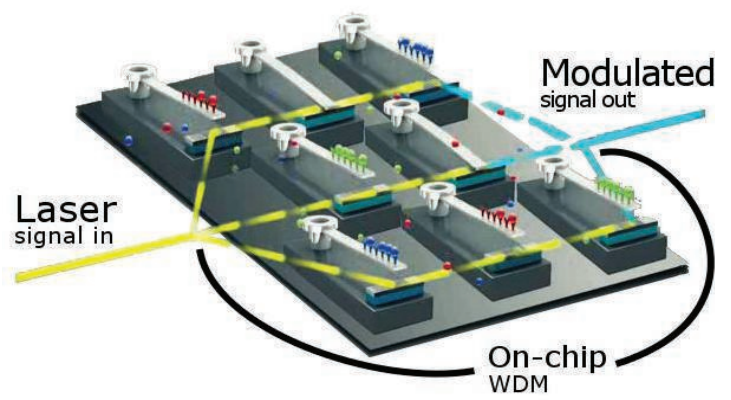

Fig. 6. Schematic of a proposed system based on on-chip WDM to address large arrays of cantilevers 
The integrated optical readout approach presented here combines the high sensitivity advantages of external optical readouts with the ability to address large arrays of integrated electronic readouts [11]. The ability to perform high-resolution measurements of large arrays of MEMS devices will have a large impact on the capabilities of future commercial solutions.

\section{Acknowledgment}

This work was performed in part at the Western Australian node of the Australian National Fabrication Facility, a company established under the National Collaborative Research Infrastructure Strategy to provide nano and micro-fabrication facilities for Australia's researchers. We acknowledge the support from the Australian Research Council, and the Office of Science of the WA State Government.

\section{References}

[1] Yole Development reports, "Status of the MEMS Industry 2014" (August 2014 edition), "MEMS \& Sensors for Mobile Phones and Tablets." see http://www.yole.fr/2014_press_releases.aspx

[2] T. P. Burg, M. Godin, S. M. Knudsen, W. Shen, G. Carlson, J. S. Foster, K. Babcock, and S. R. Manalis, "Weighing of biomolecules, single cells and single nanoparticles in fluid" Nature, 446, pp. 1066-1069, 2007; doi:10.1038/nature05741

[3] Y. T. Yang, C. Callegari, X. L. Feng, K. L. Ekinci, and M. L. Roukes, "Zeptogram-Scale Mass Sensing" Nano Lett., 6, p. 583, 2006; doi: $10.1021 / \mathrm{nl} 052134 \mathrm{~m}$

[4] F. Huber, H. P. Lang, N. Backmann, D. Rimoldi, Ch. Gerberdoi, "Direct detection of a BRAF mutation in total RNA from melanoma cells using cantilever arrays" Nature Nanotechnology 8 , 125-129, 2013; doi:10.1038/nnano.2012.263

[5] G. Wu, R. H. Datar, K. M. Hansen, T. Thundat, R. J. Cote, A. Majumdar, "Bioassay of prostatespecific antigen (PSA) using microcantilevers" Nature Biotechnology 19, 856 - 860, 2001; doi:10.1038/nbt0901-856

[6] T. P. Burg, M. Godin, S. M. Knudsen, W. Shen, G. Carlson, J. S. Foster, K. Babcock, S. R. Manalis, "Weighing of biomolecules, single cells and single nanoparticles in fluid", Nature 446, 1066-1069, 2007; doi:10.1038/nature05741

[7] Y. F. Dufrêne, "Towards nanomicrobiology using atomic force microscopy", Nature Reviews Microbiology 6, 674-680, 2008; doi:10.1038/nrmicro1948
[8] N. V. Lavrik, M. J. Sepaniak and P. G. Datskos, "Cantilever transducers as a platform for chemical and biological sensors", Rev. Sci. Instrum. 75, 2229, 2004; doi: 10.1063/1.1763252

[9] T. H. Stievater, W. S. Rabinovich, M. S. Ferraro, N. A. Papanicolaou, R. Bass, J. B. Boos, J. L. Stepnowski, R. A. McGill, " Photonic microharp chemical sensors", Opt Express; 16(4):2423-30, 2008; doi: 10.1364/OE.16.002423

[10] G. Binnig, M. Despont, U. Drechsler, W. Häberle, M. Lutwyche, P. Vettiger, H. J. Mamin, B. W. Chui, T. W. Kenny, "Ultrahigh-density atomic force microscopy data storage with erase capability" Applied Physics Letters, 74, 13291331, 1999; doi: 10.1063/1.123540

[11] G. Putrino, A. Keating, M. Martyniuk, L. Faraone, J. Dell, "Integrated Resonant Optical Readout Applicable to Large Arrays of MEMS Beams" Photonics Technology Letters, IEEE, vol.24, no.24, pp.2243,2246, 2012; doi: 10.1109/LPT.2012.2225611

[12] TA-2100 Toluene Gas Detector, see http://www.mil-ram.com/public/ ta 2100 toluene page. $\mathrm{html}$

[13] Sabah A. Abdul-Wahab, " Sick Building Syndrome: in Public Buildings and Workplaces", Springer Science \& Business Media, 2011; doi: 10.1007/978-3-642-17919-8_15

[14] Y. Miyagi, F. Shima, K. Ishido, T. Yasutake, and K. Kamikaseda, " Tremor induced by toluene misuse successfully treated by a Vim thalamotomy", J Neurol Neurosurg Psychiatry, 66(6): 794-796, 1999; doi: 10.1136/jnnp.66.6.794

[15] H. Yamaguchia, Y. Kidachib, K. Ryoyamab, "Toluene at Environmentally Relevant Low Levels Disrupts Differentiation of Astrocyte Precursor Cells", Archives of Environmental Health, Vol.57, Issue 3, 2002 doi: 10.1080/00039890209602942

[16] G. Putrino, A. Keating, M. Martyniuk, L. Faraone, J. Dell, " Model and analysis of a high sensitivity resonant optical read-out approach suitable for cantilever sensor arrays", Journal of Lightwave Technology, vol.30, no.12, pp.1863-1868, 2012, doi: 10.1109/JLT.2012.2190973

[17] G. Putrino, M. Martyniuk, A. Keating, L. Faraone, J. Dell "On-chip Read-out of Picomechanical Motion Under Ambient Conditions", Nanoscale, 7, 1927-1933, 2015; doi: 10.1039/C4NR05419A

[18] M. Urbiztondo, A. Peralta, I. Pellejero, J. Sesé, M. Pina, I. Dufour, J. Santamaría, "Detection of organic vapours with Si cantilevers coated with inorganic (zeolites) or organic (polymer) layers", Sensors and Actuators B: Chemical, vol. 171172, pp. 822-831, 2012; doi: 10.1016/j.snb.2012.05.078 